Ferrata Storti Foundation

\title{
Prognostic implications of additional genomic lesions in adult Philadelphia chromosome- positive acute lymphoblastic leukemia
}

Haematologica 2019

Volume 104(2):312-318

\section{Correspondence:}

chiaretti@bce.uniroma1.it or rfoa@bce.uniroma1.it

Received: April 26, 2018.

Accepted: August 30, 2018.

Pre-published: September 6, 2018.

doi:10.3324/haematol.2018.196055

Check the online version for the most updated information on this article, online supplements, and information on authorship \& disclosures: www.haematologica.org/content/104/2/312

\section{(C)2019 Ferrata Storti Foundation}

Material published in Haematologica is covered by copyright. All rights are reserved to the Ferrata Storti Foundation. Use of published material is allowed under the following terms and conditions:

https://creativecommons.org/licenses/by-nc/4.0/legalcode. Copies of published material are allowed for personal or internal use. Sharing published material for non-commercial purposes is subject to the following conditions:

https://creativecommons.org/licenses/by-nc/4.0/legalcode, sect. 3. Reproducing and sharing published material for commercial purposes is not allowed without permission in writing from the publisher.
Anna Lucia Fedullo, ${ }^{1 *}$ Monica Messina, ${ }^{1 *}$ Loredana Elia,${ }^{1}$ Alfonso Piciocchi, ${ }^{2}$ Valentina Gianfelici, ${ }^{1}$ Alessia Lauretti, ${ }^{1}$ Stefano Soddu, ${ }^{2}$ Maria Cristina Puzzolo, ${ }^{1}$ Clara Minotti, ${ }^{1}$ Felicetto Ferrara, ${ }^{3}$ Bruno Martino, ${ }^{4}$ Patrizia Chiusolo, ${ }^{5}$ Valeria Calafiore, ${ }^{6}$ Stefania Paolini, ${ }^{7}$ Marco Vignetti, ${ }^{1,2}$ Antonella Vitale, ${ }^{1}$ Anna Guarini, ${ }^{8}$ Robin Foà ${ }^{1 *}$ and Sabina Chiaretti ${ }^{1 *}$

${ }^{1}$ Hematology, Department of Translational and Precision Medicine, Sapienza University, Rome; ${ }^{2}$ GIMEMA Data Center, Rome; ${ }^{3}$ Division of Hematology and Stem Cell

Transplantation Unit, Cardarelli Hospital, Naples; ${ }^{4}$ Hematology Unit, Azienda Ospedaliera Bianchi Melacrino Morelli, Reggio Calabria; ${ }^{5}$ nstitute of Hematology, Catholic University, Rome; ${ }^{~ D i v i s i o n ~ o f ~ H e m a t o l o g y, ~ A O U ~ P o l i c l i n i c o, ~ U n i v e r s i t y ~ o f ~ C a t a n i a ; ~ ' " L . ~ a n d ~ A . ~ S e r a ̀ g n o l i " ~}$ Institute of Hematology, University of Bologna and ${ }^{8}$ Department of Molecular Medicine, Sapienza University, Rome, Italy

*These authors contributed equally to this work

\section{ABSTRACT}

O shed light onto the molecular basis of Philadelphia chromosome-positive acute lymphoblastic leukemia and to investigate the prognostic role of additional genomic lesions, we analyzed copy number aberrations using the Cytoscan HD Array in 116 newly diagnosed adult patients with Philadelphia chromosome-positive acute lymphoblastic leukemia enrolled in four different GIMEMA protocols, all based on a chemotherapy-free induction strategy. This analysis showed that patients with Philadelphia chromosome-positive acute lymphoblastic leukemia carry an average of 7.8 lesions/case, with deletions outnumbering gains ( $88 \%$ versus $12 \%)$. The most common deletions were those targeting IKZF1, PAX 5 and $C D K N 2 A / B$, which were detected in $84 \%, 36 \%$ and $32 \%$ of cases, respectively. Patients carrying simultaneous deletions of IKZF1 plus CDKN2A/B and/or $P A X 5$ had a significantly lower disease-free survival rate $(24.9 \%$ versus $43.3 \%$; $P=0.026)$. The only IKZF1 isoform affecting prognosis was the dominant negative one $(P=0.003)$. Analysis of copy number aberrations showed that $18 \%$ of patients harbored $M E F 2 C$ deletions, which were of two types, differing in size: the longer deletions were associated with the achievement of a complete molecular remission $(P=0.05)$ and had a favorable impact on disease-free survival (64.3\% versus $32.1 \%$ at 36 months; $P=0.031)$. These findings retained statistical significance also in multivariate analysis $(P=0.057)$. KRAS deletions, detected in $6 \%$ of cases, were associated with the achievement of a complete molecular remission $(P=0.009)$. These results indicate that in adults with Philadelphia chromosome-positive acute lymphoblastic leukemia a detailed evaluation of additional deletions - including CDKN2A/B, PAX5, IKZF1, MEF2C and KRAS - has prognostic implications and should be incorporated in the design of more personalized treatment strategies.

\section{Introduction}

The Philadelphia $(\mathrm{Ph})$ chromosome derives from the $\mathrm{t}(9 ; 22)(\mathrm{q} 34 ; \mathrm{q} 11)$ and leads to a $B C R-A B L 1$ rearrangement. ${ }^{1}$ The incidence of this chromosomal change in acute lymphoblastic leukemia (ALL) increases with age, being detected in $25 \%$ of adults and in about $50 \%$ of elderly patients. ${ }^{2}$ Prior to the advent of tyrosine kinase inhibitors, the outcome of Ph+ ALL patients was extremely poor, ${ }^{3.5}$ and the only 
possibility of a cure was allogeneic stem cell transplantation (HSCT), when feasible. ${ }^{6,7}$ The introduction of tyrosine kinase inhibitors, administered with low doses or without chemotherapy during induction, followed by consolidation chemotherapy and HSCT has markedly improved the management and outcome of adult $\mathrm{Ph}+\mathrm{ALL}$ patients, with survival rates at 5 years now approaching 50\%. . $^{817}$

Different biological features - the type of fusion transcript (i.e. p190 or p210), ${ }^{18}$ the persistence and/or reappearance of minimal residual disease (MRD), 19,20 additional genomic deletions (particularly IKZF1, and to a lesser extent $C D K N 2 A / B$ and $P A X 5^{21-24}$ ) - and the presence of mutations at relapse are associated with a worse outcome. ${ }^{25-27}$ However, a broad and refined biological algorithm that could help to optimize treatment strategies and define better whether some patients could be spared intensive treatment, including HSCT, has so far not been proposed.

To this end, in the present study we investigated copy number aberrations (CNA) in 116 newly diagnosed adult $\mathrm{Ph}+\mathrm{ALL}$ patients to identify additional molecular lesions with the aim of improving patients' stratification and management.

\section{Methods}

\section{Experimental strategy}

Bone marrow and/or peripheral blood samples from 116 patients (Table 1) with newly diagnosed Ph+ ALL enrolled in four GIMEMA (Gruppo Italiano Malattie EMatologiche dell'Adulto) trials were analyzed (Online Supplementary Table S1). The study was carried out in four phases (Online Supplementary Figure S1): (i) CNA analysis of 116 samples by Cytoscan; (ii) multiplex ligation-dependent probe amplification analysis; (iii) validation of $M E F 2 C$ deletions by digital droplet (dd) polymerase chain reaction (PCR); and (iv) MEF2C and KRAS mutational screening.

This study was approved in the context of an Associazione Italiana per la Ricerca sul Cancro (AIRC) project (10007) with Institutional Review Board number 2182/16.06.2011.

\section{Copy number aberration analysis}

CNA were analyzed using CytoScan ${ }^{\circledR} \mathrm{HD}$ Arrays (Affymetrix, Santa Clara, CA, USA) and Chromosome Analysis Suite (ChAS) software. Germline material from five paired samples was also evaluated. Recurrent deletions were validated with the Salsa MLPA P335 ALL-IKZF1 kit (MRC-Holland, Amsterdam, the Netherlands) ${ }^{28,29}$ (Online Supplementary Data). Statistical analyses on clinical correlates are described in the Online Supplementary Data.

Table 1. Patients' clinical features.

\begin{tabular}{lc} 
& Patients $(\mathbf{n = 1 1 6})$ \\
Gender (male/female) & $55 / 61$ \\
Age, years (range) & $51.1(18.9-89)$ \\
\hline Median white cell count x 10//L (range) & $25.4(1.7-597)$ \\
Median hemoglobin g/dL (range) & $9.6(4-16.3)$ \\
\hline Median platelet count x 10\%/L (range) & $50(4-333)$ \\
Fusion transcript (p190/p210/p190-210) & $70 / 29 / 16$ \\
\hline Complete molecular response* yes/no & $17 / 99$ \\
\hline
\end{tabular}

*As per protocol definition; "information missing for one patient.

\section{Digital droplet polymerase chain reaction assays}

MEF2C deletions were validated by ddPCR using the OX200 ${ }^{\mathrm{TM}}$ Droplet Digital ${ }^{\text {TM }}$ PCR System (BioRad, Hercules, CA, USA) and QuantaSoft Analysis Pro software according to the manufacturer's instructions (Online Supplementary Data).

\section{Mutational screening}

Sanger sequencing of PCR products for MEF2C and KRAS exons (Online Supplementary Table S2) was performed with the ABI-Prism 3500 sequencer (Applied Biosystem, Life Technologies, Foster City, CA, USA) (Online Supplementary Data).

\section{Results}

\section{Copy number aberration analysis}

CNA analysis revealed 7.8 aberrations/patient (range: 0 $28)$, the majority being losses ( $88 \%$ ) with only $12 \%$ gains, both spanning from whole chromosomes to focal lesions; $;^{22,23,30-32}$ no differences were recorded among trials (Figure 1A).

Gross chromosomal lesions were found in $42 \%$ of cases: the majority were losses of chromosome 7 (18.1\%), followed by monosomy of chromosome 9 or $9 p$ deletion $(9 \%)$ and gain of $1 \mathrm{q}(7.7 \%)$ (Figure 1B, Online Supplementary Table S3). Smaller deletions - limited to one to three genes and defined as focal - were found in $56 \%$ of cases.

The most frequently deleted region involved the $7 \mathrm{p} 12$ cytoband comprising IKZF1 $1^{22,23,33,34}$ which was detected in 97 cases $(84 \%)$.

PAX5 was deleted in 43 patients $(36.2 \%)$, while 37 (31.9\%) had deletions of CDKN2A/B. MLLT3, BTG1, $B T L A, C D 200$ and $R B 1$ were deleted in 30, 27, 21, 17 and 16 cases, respectively $(25.9 \%, 23 \%, 18.1 \%, 17.2 \%$, $14.6 \%$, and $13.8 \%$ ) (Figure $1 \mathrm{C}$ ).

$I K Z F 1$ deletions ( $\triangle I K Z F 1)$ occurred together with $C D K N 2 A / B$ and/or $P A X 5$ deletions in $45 / 97$ cases (46.4\%) and are defined as $\triangle I K Z F 1+C D K N 2 A$ and/or $P A X 5$ (Figure 1D): this subset displayed similar lesions to those recently described by Stanulla and colleagues. ${ }^{35}$ With regard to potential interactions, we found a significant association between IKZF1 and PAX 5 deletions $(P=0.01)$, but not with CDKN2A.

Multiplex ligation-dependent probe amplification confirmed IKZF1, PAX5, CDKN2A, BTG1, EBF1, ETV6 and $R B 1$ lesions, and allowed evaluation of $I K Z F 1$ isoforms. These isoforms were grouped into four classes: ${ }^{24,36}$ wildtype, dominant-negative (comprising $\Delta 4-7$ cases, 29.8\%), haploinsufficient (including all cases harboring a deletion that involves exon 2 - i.e. $\Delta 2-7, \Delta 2-8, \Delta 2-3, \Delta 1-3$ - or the whole gene, $57.7 \%$ ) and miscellaneous (remaining cases, $11.3 \%)$.

\section{Identification of novel lesions}

CNA analysis highlighted additional genomic lesions (Table 2, Online Supplementary Table S4). We focused in particular on MEF2C and KRAS deletions since these had prognostic significance (see below). MEF2C deletions were detected in 21 patients $(18.1 \%)$ and differed in size. According to the length of intron 1-2 losses, deletions were grouped into two categories. One category - detected in 14 cases (67\% of MEF2C deleted cases) - was characterized by a larger minimal common region $(6.2 \mathrm{~Kb})$ involving introns 1-2 and exon 2 (the first codifying exon), 
defined $\triangle M E F 2 C$-long. The other category, detected in seven patients, was smaller $(5.4 \mathrm{~Kb})$ and involved only exon 2 , and was called $\triangle M E F 2 C$-short (Figure $2 \mathrm{~A}$ ). ddPCR confirmed $M E F 2 C$ lesions in all cases. No $M E F 2 C$ mutations were identified.

$K R A S$ deletions $(\triangle K R A S)$ were detected in seven cases $(6 \%)$; the focal lesion of KRAS started in the 5' untranslated region and ended in intron 1-2, involving the first noncodifying exon (Figure $2 \mathrm{~B}$ ). The minimal common region consisted of $135 \mathrm{~Kb}$. KRAS was not affected by mutations.

\section{Impact of known and novel deletions on complete molecular response achievement and disease-free survival}

We did not find significant differences between patients with $\triangle I K Z F 1$ and $I K Z F 1$ wild-type cases with regard to achievement of complete molecular response (CMR) or disease-free survival (DFS) (Online Supplementary Figure S2). Further stratification according to IKZF1 isoforms showed that patients with the dominant-negative isoform had a lower DFS rate $(23.3 \% ; P=0.039)$ compared to patients with the other isorforms, particularly wild-type (53.3\%; $P=0.016)$ and haploinsufficient cases (40.3\%; $P=0.015)$; the DFS rate of the miscellaneous group $(34.1 \%)$ did not differ significantly from that of the dominant-negative cases (Figure 3A). These differences were not statistically significant in the overall survival analysis (Figure 3B).

We also investigated the outcome of $\triangle I K Z F 1+C D K N 2 A$ and/or $P A X 5$ cases. The CMR rate did not differ between $\triangle I K Z F 1+C D K N 2 A$ and/or $P A X 5$ and $\triangle I K Z F 1$-only cases; contrariwise, $\triangle I K Z F 1+C D K N 2 A$ and/or $P A X 5$ patients had a significantly worse DFS than $\triangle I K Z F 1$-only cases (43.3\% versus $24.9 \% ; P=0.026$ ) (Figure $3 C$ ) and an inferior overall survival (62.6\% versus $40.2 \% ; P=0.02)$ (Figure $3 \mathrm{D})$.

The presence of $\triangle M E F 2 C$-long was associated with a higher rate of CMR achievement $(P=0.05)$; this effect was not influenced by the protocol or the tyrosine kinase inhibitor used (imatinib or dasatinib). Furthermore, $\triangle M E F 2 C$-long cases were also associated with a signifi-

Table 2. Minimal common region (MCR) of identified focal lesions.

\begin{tabular}{|c|c|c|}
\hline Deleted gene & N. of patients (\%) & MCR (hg19) \\
\hline FOCAD & $29(25)$ & chr9: 20685149 - 20759956 \\
\hline CDK6 & $24(20.7)$ & chr7: 92456635 - 92266647 \\
\hline PTPRD & $21(18.1)$ & chr9: 8153932 - 8854489 \\
\hline$M E F 2 C$ & $21(18.1)$ & chr5:88122179 - 88127630 \\
\hline BTLA & $21(18.1)$ & chr3:112154702 - 112217769 \\
\hline JAK2 & $20(17.2)$ & chr9: $5123013-5234403$ \\
\hline$A D D 3$ & $18(15.5)$ & chr10: 111795029 - 111853667 \\
\hline SLX4IP & $17(14.6)$ & chr20: 10417444 - 10451891 \\
\hline CD200 & $17(14.6)$ & chr3:112054292 - 112217769 \\
\hline HBSIL & $16(13.7)$ & chr6: 135375338 - 135418257 \\
\hline ATP10A & $14(12)$ & chr15: 26055568 - 26103185 \\
\hline TOX & $8(6.9)$ & chr8:60028851 - 60110235 \\
\hline KRAS & $7(6)$ & chr12: 25402194 - 25537468 \\
\hline ARHGAP24 & $7(6)$ & chr4:86493655 - 86436188 \\
\hline$E B F 1$ & $6(5.1)$ & chr5: 158440156 - 158164599 \\
\hline LEF1 & $5(4.3)$ & chr4:109034392 - 109084557 \\
\hline MDM2 & $5(4.3)$ & chr12:69159076 - 69205287 \\
\hline TCF12 & $4(3.4)$ & chr15:57294905 - 57399047 \\
\hline$E R G$ & $2(1.7)$ & chr21:39772775 - 39788683 \\
\hline
\end{tabular}

A
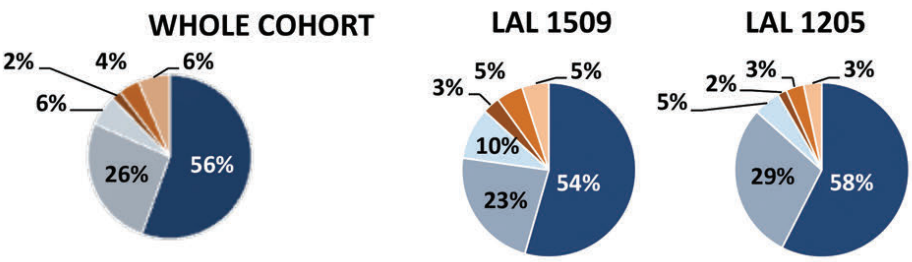

LAL 1205

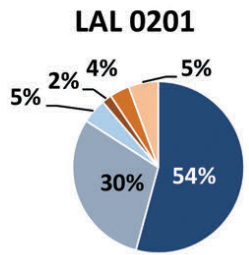

LAL 0904

- loss focal

"loss other "loss whole chromosome "gain focal

"gain other

"gain whole chromosome
B

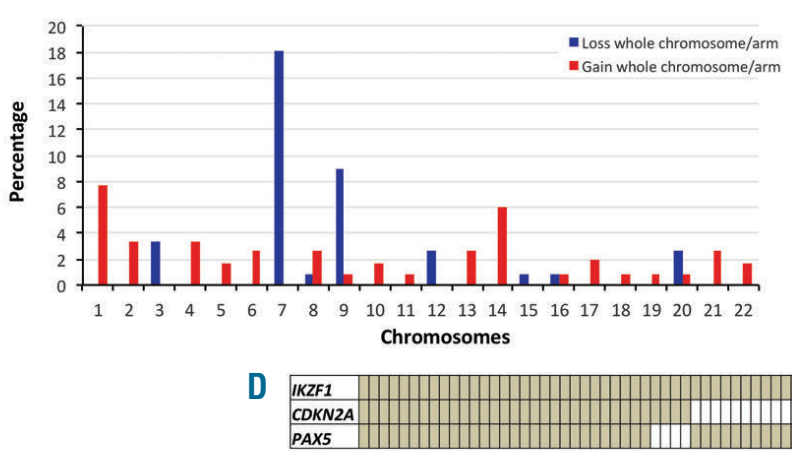
PAXY
C

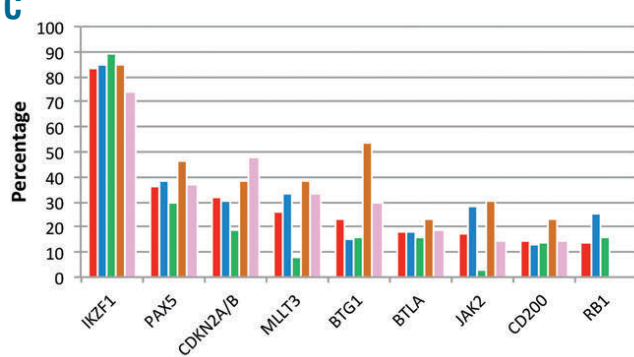

- whole cohort

= 1509

= 1205

$=0201$

$=0904$

Figure 1. Overall load and incidence of genetic lesions in Philadelphia chromosome-positive acute lymphoblastic leukemia. (A) Distribution of copy number aberrations in the whole cohort and across different protocols. (B) Percentages of gross chromosomal aberrations. (C) Percentages of deletions of known genes in the whole cohort $(\mathrm{n}=116)$ and in the different studies analyzed. (D) Heatmap of IKZF1, CDKN2A/B, and PAX5 deletions in the whole cohort. 
cantly better DFS (64.3\% versus $32.1 \%$; $P=0.031$ ) (Figure $4 \mathrm{~A})$ and overall survival $(77.9 \%$ versus $48.4 \% ; P=0.036)$ (Figure 4B).

Lastly, $\triangle K R A S$ was more frequently found in patients who obtained a CMR ( $24 \%$ versus $3 \%$; $P=0.009)$, but this finding did not have an impact on DFS.
Prognostic impact of known and novel genomic lesions in univariate and multivariate analyses

In univariate analysis, $\triangle M E F 2 C$-long and $\triangle K R A S$ had an impact on CMR achievement, while $\triangle M E F 2 C$-long and $\triangle I K Z F 1+C D K N 2 A$ and/or PAX5 influenced DFS (Table 3).

In multivariate analysis for $\mathrm{CMR}$, performed taking into
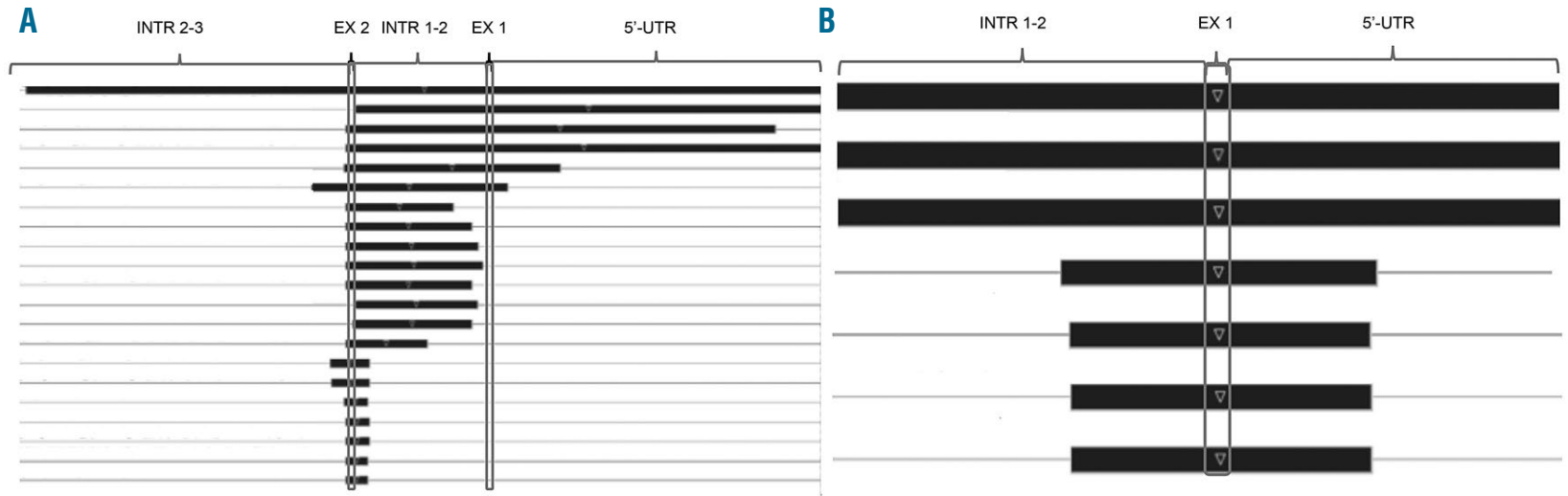

Figure 2. Representation of $\triangle M E F 2 C$ and $\triangle K R A S$. (A) Representation of $\triangle M E F 2 C$ for each patient. Lesions are ordered according to their size: one case had a deletion of the whole gene, one had a deletion that involved only exon 1 spanning from intron 1-2 to the 5' untranslated region (5'UTR), four had deletions starting from intron 2-3 and ending at 5'-UTR, thereby involving both exons 2 and 1 (the latter being an untranslated exon), 13 had lesions spanning from intron 2-3 to intron 1 2 , therefore involving exon 2 (the first codifying exon), with six of them harboring a longer intron 1-2 deletion. Lastly, two cases had deletions that involved only intron 1-2. The first 14 cases were considered as $\triangle M E F 2 C$-long and the remaining as $\triangle M E F 2 C$-short. (B) Representation of $\triangle K R A S$ for each patient. Lesions are ordered according to their size: in four cases, the deletion encompassed only KRAS itself, whereas in three it involved the short arm of chromosome 12 . INTR: intron; EX: exon; 5'UTR: 5' untranslated region.

A

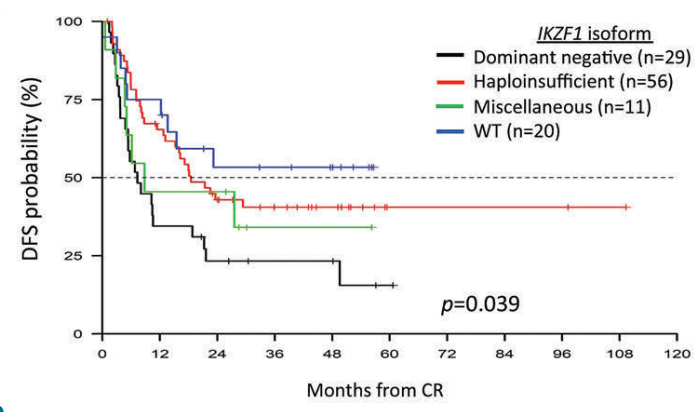

C

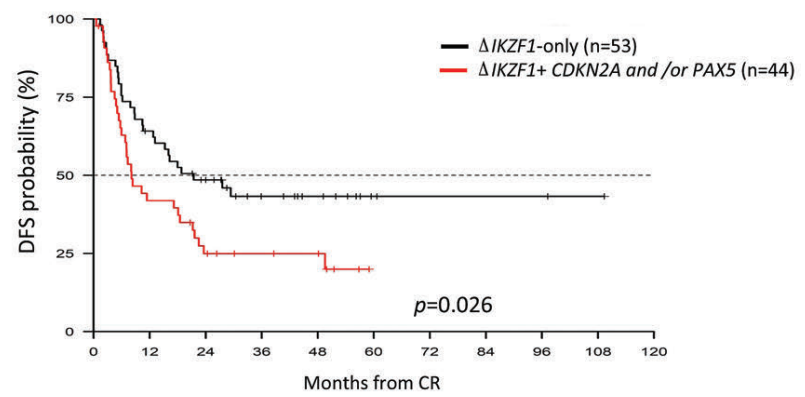

B

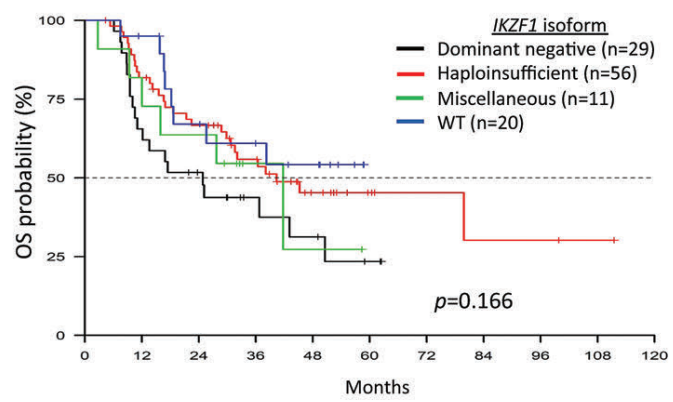

D

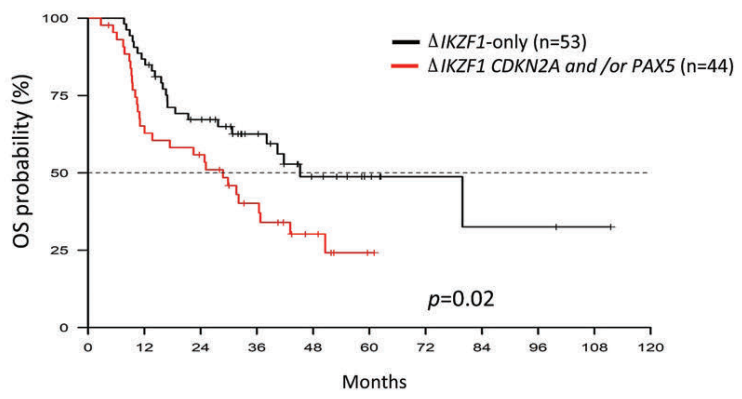

Figure 3. Survival probability curves according to IKZF1 status. (A) Disease-free survival and (B) overall survival at 36 months of patients divided according to IKZF1 isoform. (C) Disease-free survival and (D) overall survival at 36 months of $\triangle I K Z F 1$-only and $\triangle I K Z F 1+C D K N 2 A$ and/or PAX5 patients. WT: wild-type; DFS: disease-free survival; OS: overall survival; CR: complete remission. 
account white blood cell count, age, tyrosine kinase inhibitor use and the genomic lesions described above, the only factor that retained statistical significance was $\triangle K R A S(P=0.01) ;$ a trend was noted for $\triangle M E F 2 C$-long deletions $(P=0.075)$ (Table 3$)$.

In multivariate analysis for DFS, considering $\triangle M E F 2 C$ long, $\triangle I K Z F 1+C D K N 2 A$ and/or PAX5, white blood cell count and $C M R$ as variables, the factors that had a negative impact were $\triangle M E F 2 C$-long $(P=0.057)$ and white blood cell count $(P=0.05)$, while a trend towards a worse DFS was observed for $\triangle I K Z F 1+C D K N 2 A$ and/or PAX5 $(P=0.089)$ (Table 3$)$. HSCT did not affect the prognostic role of the above-mentioned lesions.

\section{Discussion}

The management of adults with Ph+ ALL currently relies on the use of first, $t^{8-10,13-16}$ second ${ }^{11,12}$ and third ${ }^{37}$ generation tyrosine kinase inhibitors, either alone ${ }^{9-12}$ or in combination with chemotherapy, ${ }^{8,13-16,37}$ followed - if feasible and necessary - by HSCT. These approaches have greatly improved the outcome of $\mathrm{Ph}+\mathrm{ALL}$ : nowadays, virtually all patients - independent of age - achieve a complete hematologic remission, coupled to a CMR in a variable proportion of cases. Nonetheless, in all reported studies the long-term outcome is in the range of $50 \%$ at 5 years; thus, additional prognosticators capable of better stratify-

Table 3. Summary of univariate and multivariate analyses for complete molecular response and disease-free survival for the factors identified.

\begin{tabular}{|c|c|c|c|c|}
\hline & $\begin{array}{l}\text { Univariate analysis for CMR } \\
\text { OR (95\% CI) }\end{array}$ & $P$ value & $\begin{array}{c}\text { Multivariate analysis for CMR } \\
\text { OR (95\% Cl) }\end{array}$ & $P$ value \\
\hline$M E F 2 C$ deletions & $0.288(0.082,1007)$ & 0.051 & $0.259(0.058,1.146)$ & 0.075 \\
\hline KRAS deletions & $0.12(0.024,0.601)$ & 0.009 & $0.068(0.009,0.529)$ & 0.01 \\
\hline White blood cell count & $0.986(0.969,1.003)$ & 0.1 & $0.986(0.966,1.007)$ & 0.188 \\
\hline Age & $1.026(0.99,1.063)$ & 0.16 & $1.028(0.985,1.072)$ & 0.205 \\
\hline Imatinib vs. dasatinib & $0.267(0.057,1.248)$ & 0.093 & $0.296(0.054,1.615)$ & 0.159 \\
\hline $\begin{array}{l}\text { Fusion protein } \\
\text { (p190 us. p210 and p190/210) }\end{array}$ & $1.247(0.421,3.693)$ & 0.691 & & \\
\hline \multirow[t]{2}{*}{$\triangle I K Z F 1+C D K N 2 A$ and/or PAX5 } & $1.600(0.599,4.581)$ & 0.381 & & \\
\hline & $\begin{array}{l}\text { Univariate analysis for DFS } \\
\text { HR (95\% CI) }\end{array}$ & $P$ value & $\begin{array}{c}\text { Multivariate analysis for DFS } \\
\text { HR (95\% })\end{array}$ & $P$ value \\
\hline$M E F 2 C$ deletions & $0.359(0.144,0.891)$ & 0.027 & $0.417(0.169-1.028)$ & 0.057 \\
\hline$\triangle I K Z F 1+C D K N 2 A$ and/or PAX5 & $1.834(1.148,2.929)$ & 0.011 & $1.608(0.930,2.781)$ & 0.089 \\
\hline White blood cell count & $1.002(1,1.004)$ & 0.065 & $1.003(1,1.006)$ & 0.050 \\
\hline Age & $1.001(0.986,1.017)$ & 0.866 & $1.01(0.995-1.028)$ & 0.180 \\
\hline CMR & $0.423(0.181-0.987)$ & 0.046 & $0.402(0.167-0.969)$ & 0.042 \\
\hline $\begin{array}{l}\text { Fusion protein } \\
\text { (p190 us. p210 and p190/210) }\end{array}$ & $0.939(0.582,1.515)$ & 0.797 & & \\
\hline Imatinib vs. dasatinib & $1.305(0.807,2.109)$ & 0.277 & & \\
\hline Allogeneic transplant & $0.682(0.362,1.284)$ & 0.23 & & \\
\hline
\end{tabular}

CMR: complete molecular response; OR: odds ratio; $95 \%$ CI: 95\% confidence interval; DFS: disease-free survival; HR: hazard ratio.

A

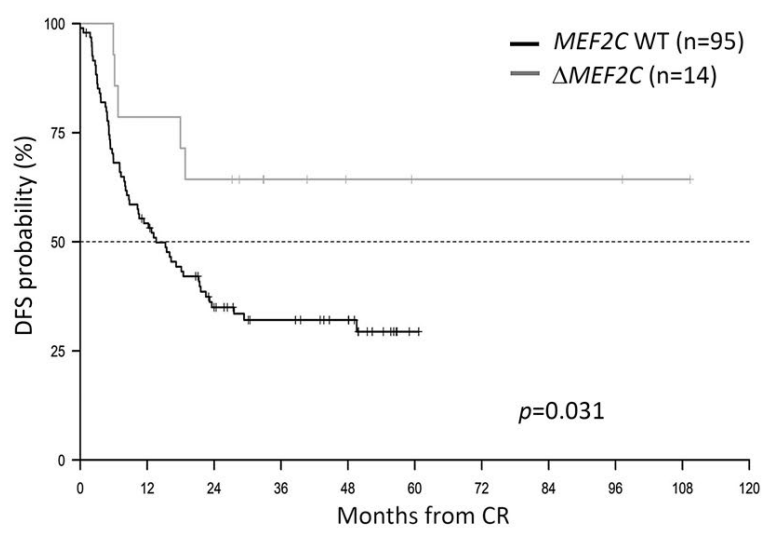

B

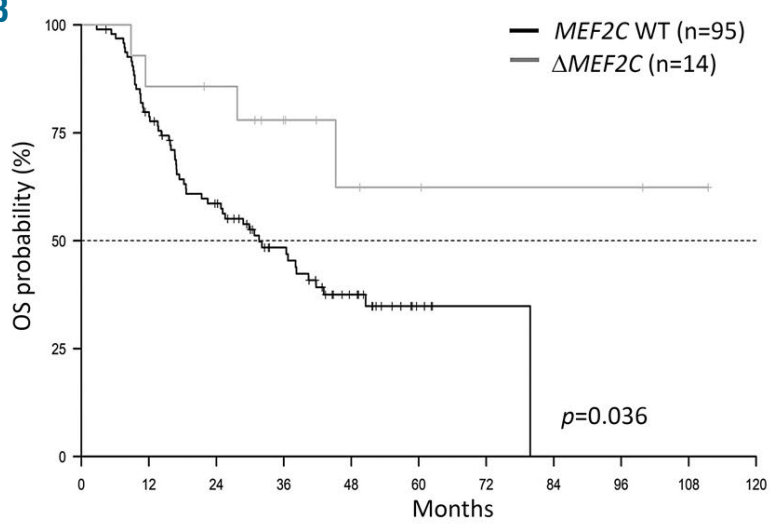

Figure 4. Survival probability curves according to MEF2C status. (A) Disease-free survival at 36 months of $\triangle M E F 2 C$ versus MEF2C wild-type patients. (B) Overall survival at 36 months of $\triangle M E F 2 C$ versus MEF2C wild-type patients. WT: wild-type; DFS: disease-free survival; OS: overall survival; CR: complete remission. 
ing patients into high- and low-risk categories are urgently needed to further optimize treatment. Moreover, another unsolved issue is whether all eligible patients should undergo HSCT, ${ }^{7,17}$ a procedure still associated with shortand long-term side effects, as well as treatment-related mortality. This is particularly important for patients who obtain a CMR.

To address these issues we sought to identify additional genomic lesions with prognostic significance in adult $\mathrm{Ph}+$ ALL using high density Cytoscan arrays. We found that adult Ph+ ALL patients carried an average of 7.8 aberrations each, with deletions outnumbering gains, in line with other ALL subsets. ${ }^{22,30,38,39}$ Macro-aberrations were identified in $48 \%$ of cases and micro-aberrations in the majority of patients: among the latter, the most frequent was $\triangle I K Z F 1$, which was detected in $84 \%$ of cases. $\triangle I K Z F 1$ has been regarded as a poor prognostic marker in both childhood and adult ALL. ${ }^{34,36,39-41}$ This finding was not, however, confirmed in our cohort: in fact, the presence of $\triangle I K Z F 1$ alone was not associated with a worse DFS. A further evaluation of the various $I K Z F 1$ isoforms showed that only the dominant-negative genotype was deleterious for outcome. In addition, patients with $\triangle I K Z F 1+C D K N 2 A$ and/or PAX5, accounting for almost half the $\triangle I K Z F 1$ cases, experienced a significantly inferior DFS $(P=0.005)$ and overall survival $(P=0.02)$, in line with previous reports on ALL in general. ${ }^{28,29,36,39,42,43}$ $\triangle I K Z F 1+C D K N 2 A$ and/or PAX5 also had a prognostic impact in multivariate analysis; survival analysis was carried out merging all cases enrolled in the different trials together in order to gain statistical significance.

Recently, studies have been focused on the presence of additional karyotypic aberrations in Ph+ ALL. ${ }^{44-48}$ These studies have highlighted that a high percentage of $\mathrm{Ph}+\mathrm{ALL}$ cases $(60-80 \%)$ harbor additional chromosomal abnormalities, with the most frequent aberrations involving chromosomes 7, 9, and 14. Patients with additional abnormalities, particularly loss of 9/9p and/or CDKN2A, have a worse outcome. These results point to the importance of screening for other molecular markers, and not only IKZF1, in agreement with our findings on $\triangle I K Z F 1+C D K N 2 A$ and/or $P A X 5$. At variance from these reports, our study also identified novel lesions that had a favorable impact on outcome. Among these, it is worth mentioning $\triangle M E F 2 C$, which occurred in $18.1 \%$ of patients and was of two sizes, a long deletion, encompassing introns 1-2 and exon 2, and a second, smaller one, involving only exon $2 . M E F 2 C$ is a transcription factor involved in B-cell survival and proliferation whose overexpression is associated with an unfavorable prognosis in T-ALL and acute myeloid leukemia. ${ }^{49-52}$ In our study, the presence of $\triangle M E F 2 C$-long was associated with achievement of a CMR $(P=0.05)$ and with a significantly better DFS compared to the remaining cases $(P=0.031)$ also in a multivariate model; as for IKZF1 deletions, survival analysis was performed merging the whole cohort because of the sample sizes. $\triangle M E F 2 C$-long was widely distributed among cases, with no association with white blood cell count, age, type of fusion protein or additional deletions. To our knowledge, this is the first report that correlates $\triangle M E F 2 C$-long with prognosis in Ph+ ALL: Martinelli et al..$^{40}$ and Mullighan et al..$^{22,41}$ described $\triangle M E F 2 C$ in Ph+ ALL, but did not demonstrate a correlation with outcome. Finally, $\triangle K R A S$ was associated with a higher rate of CMR achievement upon induction $(P=0.01)$, but not with a better DFS.

In conclusion, we show that additional genetic lesions can be found at presentation in adult Ph+ ALL patients and that these lesions have prognostic significance, with the IKZF1 dominant-negative isoform and $\triangle I K Z F 1+C D K N 2 A$ and/or PAX 5 negatively affecting outcome, and $\triangle M E F 2 C$ and $\triangle K R A S$ being instead associated with a more favorable prognosis. Screening for these genetic lesions should, therefore, be performed at the time of diagnosis for a more refined prognostic stratification, and for a more personalized and tailored management of $\mathrm{Ph}+\mathrm{ALL}$ patients.

\section{Acknowledgments}

The authors thank Associazione Italiana per la Ricerca sul Cancro (AIRC), Special Program Molecular Clinical Oncology-Extension program, $5 \times 1000$ (10007), Milan (Italy) for funding RF; Finanziamento per l'avvio alla ricerca 2015 (Sapienza University of Rome) for funding $M M$; Finanziamento Medi Progetti Universitari 2015 for funding SC (Sapienza University of Rome); and Fondazione Le Molinette Onlus, Turin (Italy).

\section{References}

1. Nowell PC, Hungerford DA. Chromosome studies on normal and leukemic human leukocytes. J Natl Cancer Inst. 1960;25:85109.

2. Chiaretti S, Vitale A, Cazzaniga G, et al. Clinico-biological features of 5202 patients with acute lymphoblastic leukemia enrolled in the Italian AIEOP and GIMEMA protocols and stratified in age cohorts. Haematologica. 2013;98(11):1702-1710

3. Dombret H, Gabert J, Boiron JM, et al. Outcome of treatment in adults Philadelphia chromosome-positive acute lymphoblastic leukemia-results of the prospective multicenter LALA-94 trial. Blood. 2002;100(7): 2357-2366.

4. Gleissner B, Gökbuget N, Bartram CR, et al. Leading prognostic relevance of the BCR$\mathrm{ABL}$ translocation in adult acute $\mathrm{B}$-lineage lymphoblastic leukemia: a prospective study of the German Multicenter Trial Group and confirmed polymerase chain reaction analysis. Blood. 2002;99(5):15361543

5. Pullarkat V, Slovak ML, Kopecky KJ, et al. Impact of cytogenetics on the outcome of adult acute lymphoblastic leukemia: results of Southwest Oncology Group 9400 study. Blood. 2008;111(5):2563-2572

6. Hunault M, Harousseau JL, Delain M, et al. Better outcome of adult acute lymphoblastic leukemia after early genoidentical allogeneic bone marrow transplantation (BMT) than after late high-dose therapy and autologous BMT: a GOELAMS trial. Blood. 2004;104(10):3028-3037.

7. Patel JN, Druhan LJ. Genetic effects on hematopoietic stem cell transplant prognosis and outcomes, more than just histocompatibility. Biol Blood Marrow Transplant. 2017;23(8):1227-1228
8. de Labarthe A, Rousselot P, Huguet-Rigal F, et al. Imatinib combined with induction or consolidation chemotherapy in patients with de novo Philadelphia chromosomepositive acute lymphoblastic leukemia: results of the GRAAPH-2003 study. Blood. 2007;109(4):1408-1413.

9. Vignetti M, Fazi P, Cimino G, et al. Imatinib plus steroids induces complete remissions and prolonged survival in elderly Philadelphia chromosome-positive patients with acute lymphoblastic leukemia without additional chemotherapy: results of the Gruppo Italiano Malattie Ematologiche dell'Adulto (GIMEMA) LAL0201-B protocol. Blood. 2007;109(9):3676-3678.

10. Chiaretti S, Vitale A, Vignetti M, et al. A sequential approach with imatinib, chemotherapy and transplant for adult $\mathrm{Ph}+$ acute lymphoblastic leukemia. Final results of the GIMEMA LAL 0904 study. Haematologica. 2016;101(12):1544-1552. 
11. Foà R, Vitale A, Vignetti M, et al. Dasatinib as first-line treatment for adult patients with Philadelphia chromosome-positive acute lymphoblastic leukemia. Blood. 2011;118 (25):6521-6528

12. Chiaretti S, Vitale A, Elia L, et al. Multicenter Total Therapy GIMEMA LAL 1509 protocol for de novo adult $\mathrm{Ph}+$ acute lymphoblastic leukemia (ALL) patients. Updated results and refined genetic-based prognostic stratification. Blood. 2015;126 (23):81.

13. Bassan R, Rossi G, Pogliani EM, et al. Chemotherapy-phased imatinib pulses improve long-term outcome of adult patients with Philadelphia chromosomepositive acute lymphoblastic leukemia: Northern Italy Leukemia Group protocol 09/00. J Clin Oncol. 2010;28(22):3644-3652.

14. Ribera JM, García $O$, Montesinos $\mathrm{P}$, et al. Treatment of young patients with Philadelphia chromosome-positive acute lymphoblastic leukaemia using increased dose of imatinib and deintensified chemotherapy before allogeneic stem cell transplantation. Br J Haematol. 2012;159 (1):78-81

15. Fielding AK, Rowe JM, Buck G, et al. UKALLXII/ECOG2993: addition of imatinib to a standard treatment regimen enhances long-term outcomes in Philadelphia positive acute lymphoblastic leukemia. Blood. 2014;123(6):843-850.

16. Chalandon $Y$, Thomas $X$, Hayette $S$, et al. Randomized study of reduced-intensity chemotherapy combined with imatinib in adults with Ph-positive acute lymphoblastic leukemia. Blood. 2015;125(24):3711-3719.

17. Litzow MR, Fielding AK, Luger SM, et al. The evolving role of chemotherapy and hematopoietic cell transplants in $\mathrm{Ph}$-positive acute lymphoblastic leukemia in adults. Bone Marrow Transplant. 2017;52(12):15921598.

18. Cimino G, Pane F, Elia L, et al. The role of $\mathrm{BCR} / \mathrm{ABL}$ isoforms in the presentation and outcome of patients with Philadelphia-positive acute lymphoblastic leukemia: a sevenyear update of the GIMEMA 0496 trial. Haematologica. 2006;91(3):377-380.

19. Lee S, Kim DW, Cho BS, et al. Impact of minimal residual disease kinetics during imatinib-based treatment on transplantation outcome in Philadelphia chromosome-positive acute lymphoblastic leukemia. Leukemia. 2012;26(11):2367-2374.

20. Ravandi F, Jorgensen JL, Thomas DA, et al. Detection of MRD may predict the outcome of patients with Philadelphia chromosomepositive ALL treated with tyrosine kinase inhibitors plus chemotherapy. Blood. 2013;122(7):1214-1221.

21. Martinelli G, Iacobucci I, Storlazzi CT, et al. IKZF1 (Ikaros) deletions in BCR-ABL1-positive acute lymphoblastic leukemia are associated with short disease-free survival and high rate of cumulative incidence of relapse: a GIMEMA AL WP report. J Clin Oncol. 2009;27(31):5202-5207.

22. Mullighan CG, Miller $\mathrm{CB}$, Radtke I, et al. BCR-ABL1 lymphoblastic leukaemia is characterized by the deletion of Ikaros. Nature. 2008;453(7191):110-114.

23. Mullighan CG. Genomic profiling of B-progenitor acute lymphoblastic leukemia. Best Pract Res Clin Haematol. 2011;24(4):489503.

24. van $\operatorname{der}$ Veer A, Zaliova M, Mottadelli F, et al. IKZF1 status as a prognostic feature in BCR-ABL1-positive childhood ALL. Blood. 2014;123(11):1691-1698

25. DeBoer R, Koval G, Mulkey F, et al. Clinical impact of ABL1 kinase domain mutations and IKZF1 deletion in adults under age 60 with Philadelphia chromosome-positive $(\mathrm{Ph}+)$ acute lymphoblastic leukemia (ALL) molecular analysis of CALGB (Alliance) 10001 and 9665. Leuk Lymphoma. 2016;57(10):2298-2306.

26. Soverini S, Vitale A, Poerio A, et al Philadelphia-positive acute lymphoblastic leukemia patients already harbor BCR-ABL kinase domain mutations at low levels at the time of diagnosis. Haematologica. 2011;96(4):552-557.

27. Soverini S, De Benedittis C, Machova Polakova K, et al. Unraveling the complexity of tyrosine kinase inhibitor-resistant populations by ultra-deep sequencing of the BCRABL kinase domain. Blood. 2013;122(9): 1634-1648.

28. Messina M, Chiaretti S, Fedullo AL, et al. Clinical significance of recurrent copy number aberrations in B-lineage acute lymphoblastic leukaemia without recurrent fusion genes across age cohorts. Br J Haematol. 2017;178(4):583-587.

29. Moorman AV, Enshaei A, Schwab C, et al. A novel integrated cytogenetic and genomic classification refines risk stratification in pediatric acute lymphoblastic leukemia. Blood. 2014;124(9):1434-1444.

30. Mullighan CG, Goorha S, Radtke I, et al Genome-wide analysis of genetic alterations in acute lymphoblastic leukaemia. Nature. 2007;446(7137):758-764.

31. Kuiper RP, Schoenmakers EF, van Reijmersdal SV, et al. High-resolution genomic profiling of childhood ALL reveals novel recurrent genetic lesions affecting pathways involved in lymphocyte differentiation and cell cycle progression. Leukemia. 2007;21(6):1258-1266

32. Safavi S, Hansson M, Karlsson K, et al. Novel gene targets detected by genomic profiling in a consecutive series of 126 adults with acute lymphoblastic leukemia. Haematologica. 2015;100(1):55-61.

33. Iacobucci I, Storlazzi CT, Cilloni D, et al. Identification and molecular characterization of recurrent genomic deletions on $7 \mathrm{p} 12$ in the IKZF1 gene in a large cohort of BCRABL1-positive acute lymphoblastic leukemia patients: on behalf of Gruppo Italiano Malattie EMatologiche dell'Adulto Acute Leukemia Working Party (GIMEMA AL WP). Blood. 2009;114(10):2159-2167.

34. Mullighan CG, Su X, Zhang J, et al. Deletion of IKZF1 and prognosis in acute lymphoblastic leukemia. N Engl J Med. 2009; 360(5):470-480.

35. Stanulla M, Dagdan E, Zaliova M, et al. IKZF1plus defines a new minimal residual disease-dependent very-poor prognostic profile in pediatric B-cell precursor acute lymphoblastic leukemia. J Clin Oncol. 2018; 36(12):1240-1249.

36. Boer JM, van der Veer A, Rizopoulos D, et al. Prognostic value of rare IKZF1 deletion in childhood B-cell precursor acute lymphoblastic leukemia: an international collaborative study. Leukemia. 2016;30(1):32-38.

37. Jabbour E, Kantariian $\mathrm{H}$, Ravandi F, et al. Combination of hyper-CVAD with ponatinib as first-line therapy for patients with Philadelphia chromosome-positive acute lymphoblastic leukaemia: a single-centre, phase 2 study. Lancet Oncol. 2015;16(15):1547-1555

38. Messina M, Chiaretti S, Wang J, et al. Prognostic and therapeutic role of targetable lesions in B-lineage acute lymphoblastic leukemia without recurrent fusion genes. Oncotarget. 2016;7(12):13886-13901.

39. Ribera J, Morgades M, Zamora L, et al.
Prognostic significance of copy number alterations in adolescent and adult patients with precursor B acute lymphoblastic leukemia enrolled in PETHEMA protocols. Cancer. 2015;121(21):3809-3817.

40. Martinelli G, Iacobucci I, Papayannidis C, et al. New targets for $\mathrm{Ph}+$ leukaemia therapy. Best Pract Res Clin Haematol. 2009;22(3): 445-454.

41. Mullighan CG, Downing JR. Genome-wide profiling of genetic alterations in acute lymphoblastic leukemia: recent insights and future directions. Leukemia. 2009;23(7): 1209-1218

42. Xu N, Li YL, Li X, et al. Correlation between deletion of the CDKN2 gene and tyrosine kinase inhibitor resistance in adult Philadelphia chromosome-positive acute lymphoblastic leukemia. J Hematol Oncol. 2016;9:40.

43. Pfeifer H, Raum K, Markovic S, et al. Genomic CDKN2A/2B deletions in adult $\mathrm{Ph}+\mathrm{ALL}$ are adverse despite allogeneic stem cell transplantation. Blood. 2018;131(13); 1464-1475.

44. Heerema NA, Harbott J, Galimberti S, et al Secondary cytogenetic aberrations in childhood Philadelphia chromosome positive acute lymphoblastic leukemia are nonrandom and may be associated with outcome Leukemia. 2004;18(4):693-702.

45. Li Y, Oiu L, Zou D, et al. Additional chromosomal abnormalities and their prognostic significance in adult Philadelphia-positive acute lymphoblastic leukemia: with or without imatinib in chemotherapy. Ann Hematol. 2009;88(11):1069-1077.

46. Short NJ, Kantarjian HM, Sasaki K,et al. Poor outcomes associated with $+\operatorname{der}(22) \mathrm{t}(9 ; 22)$ and $-9 / 9 p$ in patients with Philadelphia chromosome-positive acute lymphoblastic leukemia receiving chemotherapy plus a tyrosine kinase inhibitor. Am J Hematol. 2017;92(3):238-243

47. Seol CA, Cho YU, Jang S, et al. Prognostic significance of recurrent additional chromosomal abnormalities in adult patients with Philadelphia chromosome-positive acute lymphoblastic leukemia. Cancer Genet. 2017;216-217:29-36.

48. Motlló C, Ribera JM, Morgades M, et al Frequency and prognostic significance of additional cytogenetic abnormalities to the Philadelphia chromosome in young and older adults with acute lymphoblastic leukemia. Leuk Lymphoma. 2018;59(1):146154

49. Homminga I, Pieters R, Langerak AW, et al Integrated transcript and genome analyses reveal NKX2-1 and MEF2C as potential oncogenes in $\mathrm{T}$ cell acute lymphoblastic leukemia. Cancer Cell. 2011;19(4):484-497.

50. Zuurbier L, Gutierrez A, Mullighan CG, et al. Immature MEF2C-dysregulated T-cell leukemia patients have an early T-cell precursor acute lymphoblastic leukemia gene signature and typically have non-rearranged T-cell receptors. Haematologica. 2014;99(1): 94-102.

51. Laszlo GS, Alonzo TA, Gudgeon CJ, et al. High expression of myocyte enhancer factor 2C (MEF2C) is associated with adverse-risk features and poor outcome in pediatric acute myeloid leukemia: a report from the Children's Oncology Group. J Hematol Oncol. 2015:8:115.

52. Colomer-Lahiguera S, Pisecker M, König M et al. MEF2C-dysregulated pediatric T-cell acute lymphoblastic leukemia is associated with CDKN1B deletions and a poor response to glucocorticoid therapy. Leuk Lymphoma. 2017;58(12):2895-2904. 